\title{
Performance Analysis of SNR-Based HDAF M2M Cooperative Networks
}

\author{
Lingwei Xu, ${ }^{1}$ Hao Zhang, ${ }^{1,2}$ and T. Aaron Gulliver ${ }^{2}$ \\ ${ }^{1}$ College of Information Science and Engineering, Ocean University of China, Qingdao 266100, China \\ ${ }^{2}$ Department of Electrical and Computer Engineering, University of Victoria, Victoria, BC, Canada V8W $2 Y 2$ \\ Correspondence should be addressed to Lingwei Xu; gaomilaojia2009@163.com
}

Received 30 December 2014; Accepted 13 March 2015

Academic Editor: Adam Panagos

Copyright (c) 2015 Lingwei Xu et al. This is an open access article distributed under the Creative Commons Attribution License, which permits unrestricted use, distribution, and reproduction in any medium, provided the original work is properly cited.

\begin{abstract}
The lower bound on outage probability (OP) of mobile-to-mobile (M2M) cooperative networks over N-Nakagami fading channels is derived for SNR-based hybrid decode-amplify-forward (HDAF) relaying. The OP performance under different conditions is evaluated through numerical simulation to verify the accuracy of the analysis. These results show that the fading coefficient, number of cascaded components, relative geometric gain, and power-allocation are important parameters that influence this performance.
\end{abstract}

\section{Introduction}

Mobile-to-mobile (M2M) communications have attracted significant research interest in recent years because they are widely employed in many wireless communication systems, such as mobile ad-hoc networks and vehicle-to-vehicle networks [1]. When both the transmitter and receiver are in motion, the double-Rayleigh fading model has been found to be suitable [2]. Extending this model by characterizing the fading between each pair of transmit and receive antennas as Nakagami, the double-Nakagami fading model has also been considered [3]. The $N$-Nakagami distribution was introduced in [4] as the product of $N$ statistically independent, but not necessarily identically distributed, Nakagami random variables.

Cooperative diversity has been proposed for the high data-rate coverage required in $\mathrm{M} 2 \mathrm{M}$ communication networks. Using amplify-and-forward (AF) relaying, the pairwise error probability (PEP) was investigated in [5] for cooperative intervehicular communication (IVC) systems over double-Nakagami fading channels. In [6], the exact symbol error rate (SER) and asymptotic SER expressions were derived for a M2M system with decode-and-forward (DF) relaying using the well-known moment generating function (MGF) approach over double-Nakagami fading channels. Symbol error probability (SEP) expressions were obtained in [7] using this approach for multiple-mobile-relay $\mathrm{M} 2 \mathrm{M}$ systems employing adaptive DF (ADF) relaying and fixed-gain AF (FAF) relaying over double-Nakagami fading channels.

In [8], a novel cooperative diversity protocol called hybrid decode-amplify-forward (HDAF) was proposed. This protocol combines AF and ADF relaying. When the quality of the received signal is sufficient, the relay performs ADF relaying; otherwise AF relaying is employed instead of remaining silent. However, only the SEP performance was considered, and the analysis is based on the assumption that the relay can determine whether each received symbol is correctly detected or not, which is not practical in real systems. To provide a practical HDAF protocol, in [9] the forwarding decisions at the relay were based on the signal-to-noise ratio (SNR) of the received signal. An SNR-based HDAF relaying scheme was also proposed. Further, closed-form expressions for the bit error probability of SNR-based HDAF relaying over independent nonidentical flat Rayleigh fading channels with maximum ratio combining (MRC) were derived.

To the best of our knowledge, the outage probability (OP) performance of SNR-based HDAF relaying M2M cooperative networks over $N$-Nakagami fading channels has not been considered in the literature. Thus in this paper, we present the analysis for the $N$-Nakagami case which subsumes the double-Nakagami results in [5-7] as special cases. Exact 
OP expressions are derived for SNR-based HDAF relaying over $N$-Nakagami fading channels. The influence of the fading coefficient, number of cascaded components, relative geometric gain, and power-allocation on the OP performance is investigated.

The remainder of this paper is organized as follows. The SNR-based HDAF relaying model is presented in Section 2. Section 3 provides exact OP expressions for SNR-based HDAF relaying. Monte Carlo simulation results are presented in Section 4. Finally, some concluding remarks are given in Section 5.

\section{System Model}

We consider a three node cooperation model with a mobile source (MS), a mobile relay (MR), and a mobile destination (MD). These nodes operate in half-duplex mode and are equipped with a single pair of transmit and receive antennas.

According to [5], let $d_{\mathrm{SD}}, d_{\mathrm{SR}}$, and $d_{\mathrm{RD}}$ represent the MS to $\mathrm{MD}, \mathrm{MS}$ to $\mathrm{MR}$, and MR to MD links, respectively. Assuming the path loss between the MS and MD to be unity, the relative gain of the MS to MR and MR to MD links is defined as $G_{\mathrm{SR}}=\left(d_{\mathrm{SD}} / d_{\mathrm{SR}}\right)^{v}$ and $G_{\mathrm{RD}}=\left(d_{\mathrm{SD}} / d_{\mathrm{RD}}\right)^{v}$, respectively, where $v$ is the path loss coefficient [10]. Further, define the relative geometric gain $\mu=G_{\mathrm{SR}} / G_{\mathrm{RD}}$ (in $\mathrm{dB}$ ), which is determined by the location of the relay with respect to the source and destination [5]. When the relay is close to the destination, the value of $\mu$ is negative. When the relay is close to the source, the value of $\mu$ is positive. When the relay has the same distance to the source and destination nodes, $\mu$ is $0 \mathrm{~dB}$.

Let $h=h_{k}, k \in\{\mathrm{SD}, \mathrm{SR}, \mathrm{RD}\}$, represent the complex channel coefficients of the MS to MD, MS to MR, and MR to MD links, respectively, which follow an $N$-Nakagami distribution. Therefore $h$ is the product of $N$ statistically independent, but not necessarily identically distributed, independent random variables:

$$
h=\prod_{i=1}^{N} a_{i}
$$

where $N$ is the number of cascaded components and $a_{i}$ is a Nakagami distributed random variable with probability density function (PDF):

$$
f(a)=\frac{2 m^{m}}{\Omega^{m} \Gamma(m)} a^{2 m-1} \exp \left(-\frac{m}{\Omega} a^{2}\right),
$$

where $\Gamma(\cdot)$ is the Gamma function, $m$ is the fading coefficient, and $\Omega$ is the scaling factor.

The PDF of $h$ is given by [4]:

$$
f_{h}(h)=\frac{2}{h \prod_{i=1}^{N} \Gamma\left(m_{i}\right)} G_{0, N}^{N, 0}\left[\left.h^{2} \prod_{i=1}^{N} \frac{m_{i}}{\Omega_{i}}\right|_{m_{1}, \ldots, m_{N}} ^{-}\right],
$$

where $G[\cdot]$ is Meijer's $G$-function.
Let $y=\left|h_{k}\right|^{2}, k \in\{\mathrm{SD}, \mathrm{SR}, \mathrm{RD}\}$, so that $y_{\mathrm{SD}}=\left|h_{\mathrm{SD}}\right|^{2}$, $y_{\mathrm{SR}}=\left|h_{\mathrm{SR}}\right|^{2}$, and $y_{\mathrm{RD}}=\left|h_{\mathrm{RD}}\right|^{2}$. The corresponding cumulative density function (CDF) of $y$ can be derived as [4]

$$
F_{y}(y)=\frac{1}{\prod_{i=1}^{N} \Gamma\left(m_{i}\right)} G_{1, N+1}^{N, 1}\left[\left.y \prod_{i=1}^{N} \frac{m_{i}}{\Omega_{i}}\right|_{m_{1}, \ldots, m_{N}, 0} ^{1}\right] .
$$

By taking the first derivative of (4) with respect to $y$, the corresponding PDF can be obtained as [4]:

$$
f_{y}(y)=\frac{1}{y \prod_{i=1}^{N} \Gamma\left(m_{i}\right)} G_{0, N}^{N, 0}\left[\left.y \prod_{i=1}^{N} \frac{m_{i}}{\Omega_{i}}\right|_{m_{1}, \ldots, m_{N}} ^{-}\right] .
$$

Communication in an SNR-based hybrid decodeamplify-forward (HDAF) relaying system can be described as follows. During the first time slot, the MS broadcasts to the MD and relay. The received signals $r_{\mathrm{SD}}$ and $r_{\mathrm{SR}}$ at the MD and MR can then be written as

$$
\begin{gathered}
r_{\mathrm{SD}}=\sqrt{K E} h_{\mathrm{SD}} x+n_{D}, \\
r_{\mathrm{SR}}=\sqrt{G_{\mathrm{SR}} K E} h_{\mathrm{SR}} x+n_{\mathrm{SR}},
\end{gathered}
$$

where $x$ denotes the transmitted signal and $n_{D}$ and $n_{\mathrm{SR}}$ are zero-mean complex Gaussian random variables with variance $N_{0} / 2$ per dimension. Here, $E$ is the total energy used by both the source and the relay during the two time slots. $K$ is the power-allocation parameter that controls the fraction of power reserved for the broadcast phase. If $K=0.5$, equal power allocation (EPA) is used.

During the second time slot, by comparing $\gamma_{\mathrm{SR}}$ with a threshold $\gamma_{T}$, the MR decides whether DF or AF cooperation is utilized to forward the received signal. $\gamma_{\mathrm{SR}}$ denotes the instantaneous SNR of the MS to MR link. If $\gamma_{\mathrm{SR}}>\gamma_{T}$, the MR decodes the received signal and generates a signal $x_{1}$ which is forwarded to the MD. With DF cooperation, the received signal at the MD is given by

$$
r_{\mathrm{RD}}=\sqrt{(1-K) G_{\mathrm{RD}} E} h_{\mathrm{RD}} x_{1}+n_{\mathrm{RD}},
$$

where $n_{\mathrm{RD}}$ is a conditionally zero-mean complex Gaussian random variable with variance $N_{0} / 2$ per dimension.

If selection combining (SC) is used at the $\mathrm{MD}$, the output SNR is

$$
\gamma_{\mathrm{SC}}=\max \left(\gamma_{\mathrm{SD}}, \gamma_{\mathrm{RD}}\right)
$$

where

$$
\begin{gathered}
\gamma_{\mathrm{SD}}=\frac{K\left|h_{\mathrm{SD}}\right|^{2} E}{N_{0}}=K\left|h_{\mathrm{SD}}\right|^{2} \bar{\gamma} \\
\gamma_{\mathrm{RD}}=\frac{(1-K) G_{\mathrm{RD}}\left|h_{\mathrm{RD}}\right|^{2} E}{N_{0}}=(1-K) G_{\mathrm{RD}}\left|h_{\mathrm{RD}}\right|^{2} \bar{\gamma} .
\end{gathered}
$$

If $\gamma_{\mathrm{SR}}<\gamma_{T}$, the MR amplifies and forwards the signal to the MD. Based on the AF cooperation protocol, the received signal at the $\mathrm{MD}$ is then given by

$$
r_{\mathrm{RD}}=\sqrt{c E} h_{\mathrm{SR}} h_{\mathrm{RD}} x+n_{\mathrm{RD}} \text {, }
$$


where

$$
c=\frac{K(1-K) G_{\mathrm{SR}} G_{\mathrm{RD}} E / N_{0}}{1+K G_{\mathrm{SR}}\left|h_{\mathrm{SR}}\right|^{2} E / N_{0}+(1-K) G_{\mathrm{RD}}\left|h_{\mathrm{RD}}\right|^{2} E / N_{0}} .
$$

If selection combining (SC) is employed at the MD, the output SNR at the MD is

$$
\gamma_{\mathrm{SCC}}=\max \left(\gamma_{\mathrm{SD}}, \gamma_{\mathrm{SRD}}\right)
$$

where

$$
\begin{gathered}
\gamma_{\mathrm{SRD}}=\frac{\gamma_{\mathrm{SR}} \gamma_{\mathrm{RD}}}{1+\gamma_{\mathrm{SR}}+\gamma_{\mathrm{RD}}}, \\
\gamma_{\mathrm{SR}}=\frac{K G_{\mathrm{SR}}\left|h_{\mathrm{SR}}\right|^{2} E}{N_{0}}=K G_{\mathrm{SR}}\left|h_{\mathrm{SR}}\right|^{2} \bar{\gamma}
\end{gathered}
$$

\section{OP of M2M Cooperative Networks}

In this section, the OP for $\mathrm{M} 2 \mathrm{M}$ cooperative networks is evaluated. The output SNR at the MD is

$$
\begin{aligned}
P_{\text {out }} & =\operatorname{Pr}\left(\gamma_{\mathrm{SR}}>\gamma_{T}, \gamma_{\mathrm{SC}}<\gamma_{\mathrm{th}}\right)+\operatorname{Pr}\left(\gamma_{\mathrm{SR}}<\gamma_{T}, \gamma_{\mathrm{SCC}}<\gamma_{\mathrm{th}}\right) \\
& =I_{1}+I_{2},
\end{aligned}
$$

where $\gamma_{\text {th }}$ is the threshold.

Next, $I_{1}$ and $I_{2}$ are evaluated. First, consider $I_{1}$. As $\gamma_{\mathrm{SD}}$, $\gamma_{\mathrm{SR}}$, and $\gamma_{\mathrm{RD}}$ are mutually independent random variables, $I_{1}$ can be simplified as follows:

$$
\begin{aligned}
I_{1} & =\operatorname{Pr}\left(\gamma_{\mathrm{SR}}>\gamma_{T}, \gamma_{\mathrm{SC}}<\gamma_{\mathrm{th}}\right) \\
& =\operatorname{Pr}\left(\gamma_{\mathrm{SR}}>\gamma_{T}\right) \operatorname{Pr}\left(\gamma_{\mathrm{SD}}<\gamma_{\mathrm{th}}\right) \operatorname{Pr}\left(\gamma_{\mathrm{RD}}<\gamma_{\mathrm{th}}\right) \\
& =\left(1-\operatorname{Pr}\left(\gamma_{\mathrm{SR}} \leq \gamma_{T}\right)\right) \operatorname{Pr}\left(\gamma_{\mathrm{SD}}<\gamma_{\mathrm{th}}\right) \operatorname{Pr}\left(\gamma_{\mathrm{RD}}<\gamma_{\mathrm{th}}\right) \\
& =\left(1-F_{\gamma_{\mathrm{SR}}}\left(\gamma_{T}\right)\right) F_{\gamma_{\mathrm{SD}}}\left(\gamma_{\mathrm{th}}\right) F_{\gamma_{\mathrm{RD}}}\left(\gamma_{\mathrm{th}}\right) .
\end{aligned}
$$

The CDF of $\gamma_{\mathrm{SD}}$ can be expressed as

$$
F_{\gamma_{\mathrm{SD}}}(r)=\frac{1}{\prod_{i=1}^{N} \Gamma\left(m_{i}\right)} G_{1, N+1}^{N, 1}\left[\left.\frac{r}{\overline{\gamma_{\mathrm{SD}}}} \prod_{i=1}^{N} \frac{m_{i}}{\Omega_{i}}\right|_{m_{1}, \ldots, m_{N}, 0} ^{1}\right],
$$

where

$$
\overline{\gamma_{\mathrm{SD}}}=K \bar{\gamma}
$$

The CDF of $\gamma_{\mathrm{SR}}$ is then

$$
F_{\gamma_{\mathrm{SR}}}(r)=\frac{1}{\prod_{t=1}^{N} \Gamma\left(m_{t}\right)} G_{1, N+1}^{N, 1}\left[\left.\frac{r}{\overline{\gamma_{\mathrm{SR}}}} \prod_{t=1}^{N} \frac{m_{t}}{\Omega_{t}}\right|_{m_{1}, \ldots, m_{N}, 0} ^{1}\right],
$$

where

$$
\overline{\gamma_{\mathrm{SR}}}=K G_{\mathrm{SR}} \bar{\gamma}
$$

The CDF of $\gamma_{\mathrm{RD}}$ is given by

$$
F_{\gamma_{\mathrm{RD}}}(r)=\frac{1}{\prod_{t t=1}^{N} \Gamma\left(m_{t t}\right)} G_{1, N+1}^{N, 1}\left[\left.\frac{r}{\overline{\gamma_{\mathrm{RD}}}} \prod_{t t=1}^{N} \frac{m_{t t}}{\Omega_{t t}}\right|_{m_{1}, \ldots, m_{N}, 0} ^{1}\right],
$$

where

$$
\overline{\gamma_{\mathrm{RD}}}=(1-K) G_{\mathrm{RD}} \bar{\gamma} \text {. }
$$

As $\gamma_{\mathrm{SR}}$ and $\gamma_{\mathrm{SRD}}$ are not mutually independent random variables, $I_{2}$ can be expressed as

$$
I_{2}=\operatorname{Pr}\left(\gamma_{\mathrm{SD}}<\gamma_{\mathrm{th}}\right) \operatorname{Pr}\left(\gamma_{\mathrm{SR}}<\gamma_{\mathrm{T}}, \gamma_{\mathrm{SRD}}<\gamma_{\mathrm{th}}\right)
$$

It is difficult to obtain the OP using $\gamma_{\mathrm{SRD}}$, but a lower bound can be obtained. This provides a lower bound on the OP of a M2M cooperative network.

Using the well-known inequality in [11], $\gamma_{\mathrm{SRD}}$ can be approximated as

$$
\gamma_{\mathrm{SRD}}<\gamma_{u p}=\min \left(\gamma_{\mathrm{SR}}, \gamma_{\mathrm{RD}}\right)
$$

This approximation is used in the OP derivation instead of $\gamma_{\mathrm{SRD}}$ since it is more tractable analytically. $I_{2}$ can be approximated as

$$
\begin{aligned}
I I_{2} & =\operatorname{Pr}\left(\gamma_{\mathrm{SD}}<\gamma_{\mathrm{th}}\right) \operatorname{Pr}\left(\gamma_{\mathrm{SR}}<\gamma_{\mathrm{T}}, \gamma_{u p}<\gamma_{\mathrm{th}}\right) \\
& =\operatorname{Pr}\left(\gamma_{\mathrm{SD}}<\gamma_{\mathrm{th}}\right) \operatorname{Pr}\left(\gamma_{\mathrm{SR}}<\gamma_{\mathrm{T}}, \min \left(\gamma_{\mathrm{SR}}, \gamma_{\mathrm{RD}}\right)<\gamma_{\mathrm{th}}\right) .
\end{aligned}
$$

Case 1. It is obvious that $\min \left(\gamma_{\mathrm{SR}}, \gamma_{\mathrm{RD}}\right) \leq \gamma_{\mathrm{SR}}$, so if $\gamma_{\mathrm{SR}}<\gamma_{T}$, then $\min \left(\gamma_{\mathrm{SR}}, \gamma_{\mathrm{RD}}\right)<\gamma_{T}$. If $\gamma_{\mathrm{th}}>\gamma_{T}$, then $\min \left(\gamma_{\mathrm{SR}}, \gamma_{\mathrm{RD}}\right)<\gamma_{\mathrm{th}}$. Therefore, (24) can be expressed as

$$
I I_{2}=\operatorname{Pr}\left(\gamma_{\mathrm{SD}}<\gamma_{\mathrm{th}}\right) \operatorname{Pr}\left(\gamma_{\mathrm{SR}}<\gamma_{T}\right)=F_{\gamma_{\mathrm{SD}}}\left(\gamma_{\mathrm{th}}\right) F_{\gamma_{\mathrm{SR}}}\left(\gamma_{T}\right)
$$

Substituting (15) and (25) into (14), the lower bound on the OP of the M2M cooperative network is

$$
\begin{aligned}
P_{\text {lower }}= & \left(1-F_{\gamma_{\mathrm{SR}}}\left(\gamma_{T}\right)\right) F_{\gamma_{\mathrm{SD}}}\left(\gamma_{\mathrm{th}}\right) F_{\gamma_{\mathrm{RD}}}\left(\gamma_{\mathrm{th}}\right) \\
& +F_{\gamma_{\mathrm{SD}}}\left(\gamma_{\mathrm{th}}\right) F_{\gamma_{\mathrm{SR}}}\left(\gamma_{T}\right) .
\end{aligned}
$$


Case 2. If $\gamma_{\mathrm{th}}<\gamma_{T}$, using the Total Probability Theorem [12, Equation (2.36)], we have

$$
\begin{aligned}
Q= & \operatorname{Pr}\left(\gamma_{\mathrm{SR}}<\gamma_{T}, \min \left(\gamma_{\mathrm{SR}}, \gamma_{\mathrm{RD}}\right)<\gamma_{\mathrm{th}}\right) \\
= & \operatorname{Pr}\left(\gamma_{\mathrm{SR}}<\gamma_{T}, \gamma_{\mathrm{SR}}>\gamma_{\mathrm{RD}}, \gamma_{\mathrm{RD}}<\gamma_{\mathrm{th}}\right) \\
& +\operatorname{Pr}\left(\gamma_{\mathrm{SR}}<\gamma_{T}, \gamma_{\mathrm{SR}}<\gamma_{\mathrm{RD}}, \gamma_{\mathrm{SR}}<\gamma_{\mathrm{th}}\right) \\
= & \operatorname{Pr}\left(\gamma_{\mathrm{RD}}<\gamma_{\mathrm{SR}}<\gamma_{T}, \gamma_{\mathrm{RD}}<\gamma_{\mathrm{th}}\right) \\
& +\operatorname{Pr}\left(\gamma_{\mathrm{SR}}<\gamma_{\mathrm{RD}}, \gamma_{\mathrm{SR}}<\gamma_{\mathrm{th}}\right) \\
= & \int_{0}^{\gamma_{\mathrm{th}}} \int_{\gamma_{\mathrm{RD}}}^{\gamma_{T}} f_{\gamma_{\mathrm{SR}}}(y) d y f_{\gamma_{\mathrm{RD}}}(z) d z \\
& +\int_{0}^{\gamma_{\mathrm{th}}} \int_{\gamma_{\mathrm{SR}}}^{\infty} f_{\gamma_{\mathrm{RD}}}(y) d y f_{\gamma_{\mathrm{SR}}}(z) d z \\
= & \int_{0}^{\gamma_{\mathrm{th}}}\left(\int_{0}^{\gamma_{T}} f_{\gamma_{\mathrm{SR}}}(y) d y-\int_{0}^{\gamma_{\mathrm{RD}}} f_{\gamma_{\mathrm{SR}}}(y) d y\right) f_{\gamma_{\mathrm{RD}}}(z) d z \\
& +\int_{0}^{\gamma_{\mathrm{th}}}\left(\int_{0}^{\infty} f_{\gamma_{\mathrm{RD}}}(y) d y-\int_{0}^{\gamma_{\mathrm{SR}}} f_{\gamma_{\mathrm{RD}}}(y) d y\right) f_{\gamma_{\mathrm{SR}}}(z) d z .
\end{aligned}
$$

From the Appendix, (24) can be simplified as

$$
I I_{2}=F_{\gamma_{\mathrm{SD}}}\left(\gamma_{\mathrm{th}}\right) Q \text {. }
$$

Substituting (15) and (28) into (14), the lower bound on the OP of the M2M cooperative network is

$$
P_{\text {lower }}=\left(1-F_{\gamma_{\mathrm{SR}}}\left(\gamma_{T}\right)\right) F_{\gamma_{\mathrm{SD}}}\left(\gamma_{\mathrm{th}}\right) F_{\gamma_{\mathrm{RD}}}\left(\gamma_{\mathrm{th}}\right)+F_{\gamma_{\mathrm{SD}}}\left(\gamma_{\mathrm{th}}\right) Q \text {. }
$$

\section{Numerical Results}

In this section, numerical results are presented to illustrate and verify the $\mathrm{OP}$ analysis given in the previous sections.

Figure 1 presents the OP performance of a M2M cooperative network when $\gamma_{\text {th }}>\gamma_{T}$. The relative geometric gain is $\mu=0 \mathrm{~dB}$, the power-allocation parameter is $K=0.5$, and the thresholds are $\gamma_{\text {th }}=4 \mathrm{~dB}$ and $\gamma_{T}=2 \mathrm{~dB}$. The following cases are considered based on the number of cascaded components $N$ and the fading coefficient $m$.

Scenario 1. $m_{\mathrm{SD}}=1, m_{\mathrm{SR}}=1, m_{\mathrm{RD}}=1$ and $N_{\mathrm{SD}}=2, N_{\mathrm{SR}}=$ $N_{\mathrm{RD}}=2$.

Scenario 2. $m_{\mathrm{SD}}=2, m_{\mathrm{SR}}=2, m_{\mathrm{RD}}=2$ and $N_{\mathrm{SD}}=2, N_{\mathrm{SR}}=$ $N_{\mathrm{RD}}=2$.

Figure 1 shows that the numerical simulation results coincide with the theoretical results, which verifies the accuracy of the analysis. As the SNR increases, the OP performance is improved. For example, in Case 2, when $\mathrm{SNR}=16 \mathrm{~dB}$, the $\mathrm{OP}$ is $3 \times 10^{-3}$, and when $\mathrm{SNR}=20 \mathrm{~dB}$, the OP is decreased to $2 \times 10^{-4}$. The OP performance is also improved with a larger fading coefficient $m$. When SNR $=12 \mathrm{~dB}$ and $m=1$, the OP is $1.8 \times 10^{-1}$, and when $m=2$, the OP is $3 \times 10^{-2}$.

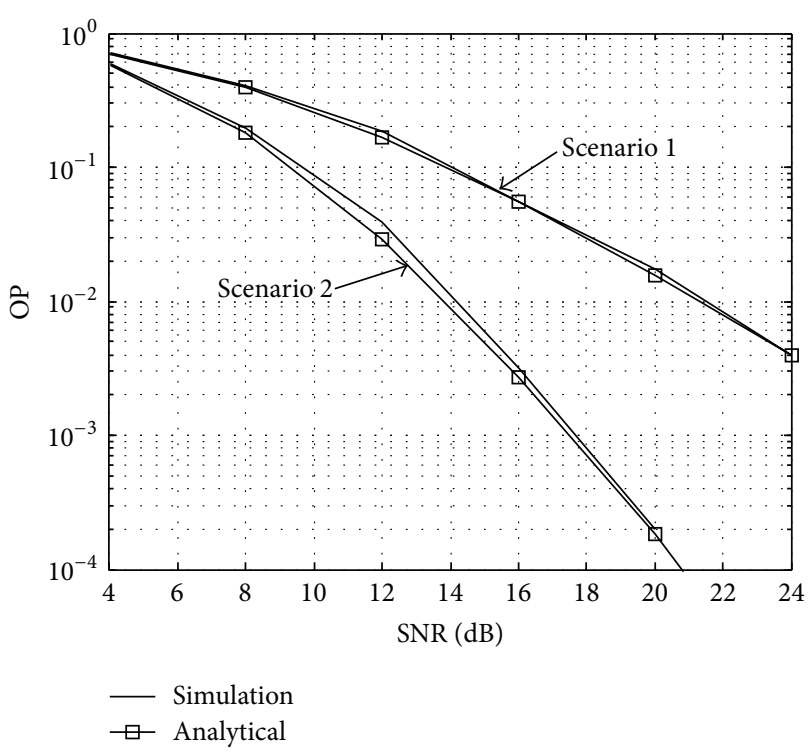

FIGURE 1: The OP performance over $N$-Nakagami fading channels when $\gamma_{\text {th }}>\gamma_{T}$.

Figure 2 presents the OP performance of the M2M cooperative network when $\gamma_{\text {th }}<\gamma_{T}$. The relative geometric gain is $\mu=0 \mathrm{~dB}$, the power-allocation parameter is $K=0.5$, and the thresholds are $\gamma_{\mathrm{th}}=2 \mathrm{~dB}$ and $\gamma_{T}=4 \mathrm{~dB}$. The following cases are considered based on the number of cascaded components $N$ and the fading coefficient $m$.

Scenario 1. $m_{\mathrm{SD}}=1, m_{\mathrm{SR}}=1, m_{\mathrm{RD}}=1$ and $N_{\mathrm{SD}}=2, N_{\mathrm{SR}}=$ $N_{\mathrm{RD}}=2$.

Scenario 2. $m_{\mathrm{SD}}=2, m_{\mathrm{SR}}=2, m_{\mathrm{RD}}=2$ and $N_{\mathrm{SD}}=2, N_{\mathrm{SR}}=$ $N_{\mathrm{RD}}=2$.

Scenario 3. $m_{\mathrm{SD}}=3, m_{\mathrm{SR}}=3, m_{\mathrm{RD}}=3$ and $N_{\mathrm{SD}}=2, N_{\mathrm{SR}}=$ $N_{\mathrm{RD}}=2$.

Figure 2 shows that the numerical simulation results coincide with the theoretical results, which verifies the analysis. As the SNR increases, the OP performance improves, as expected. For example, in Case 2, when $S N R=12 \mathrm{~dB}$, the OP is $1.5 \times 10^{-2}$, but when $\mathrm{SNR}=16 \mathrm{~dB}$, the OP is $1 \times 10^{-3}$. The OP performance also improves if the fading coefficient $m$ is increased. For example, when SNR $=12 \mathrm{~dB}$ and $m=1$, the OP is $1 \times 10^{-1}$, but when $m=2$, the OP is $1.5 \times 10^{-2}$, and when $m=3$, the OP is $2 \times 10^{-3}$.

Figure 3 presents the effect of the power-allocation parameter $K$ on the OP performance of the M2M cooperative network over $N$-Nakagami fading channels versus the SNR. The number of cascaded components is $N=2$, and the fading coefficient is $m=2$. The relative geometric gain is $\mu=0 \mathrm{~dB}$, and the thresholds are $\gamma_{\text {th }}=4 \mathrm{~dB}$ and $\gamma_{T}=2 \mathrm{~dB}$. These results show that the OP performance is improved when the SNR is increased. For example, when $K=0.6$ and $\mathrm{SNR}=10 \mathrm{~dB}$, the $\mathrm{OP}$ is $1.8 \times 10^{-2}$, when $\mathrm{SNR}=15 \mathrm{~dB}$, the $\mathrm{OP}$ is $9 \times 10^{-4}$, and when $\mathrm{SNR}=20 \mathrm{~dB}$, the OP is $2.5 \times 10^{-5}$. For $\mathrm{SNR}=10 \mathrm{~dB}$, the optimal value of $K$ is approximately 0.5 , for $\mathrm{SNR}=15 \mathrm{~dB}$, the 


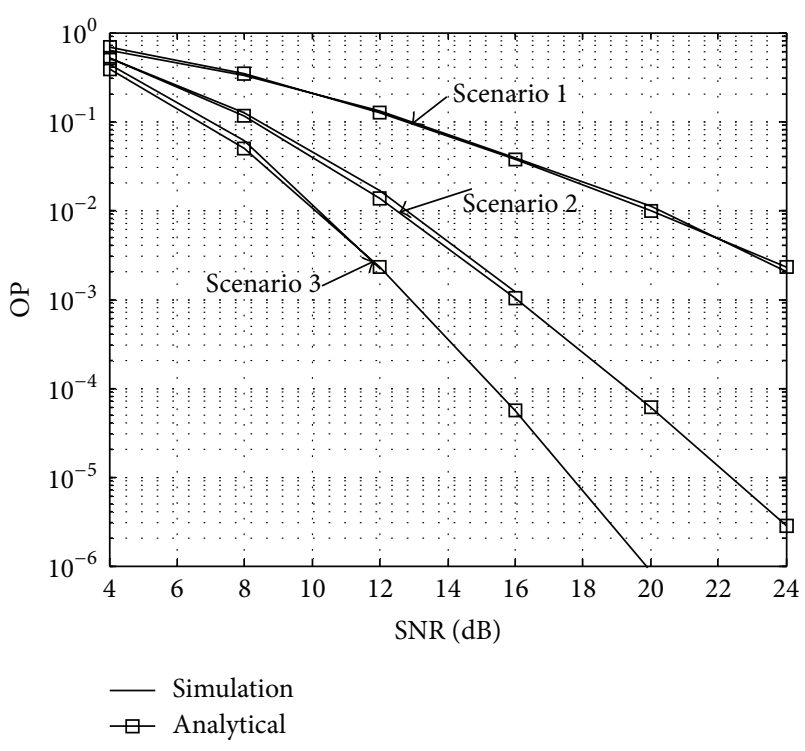

FIgURE 2: The OP performance over $N$-Nakagami fading channels when $\gamma_{\text {th }}<\gamma_{T}$.

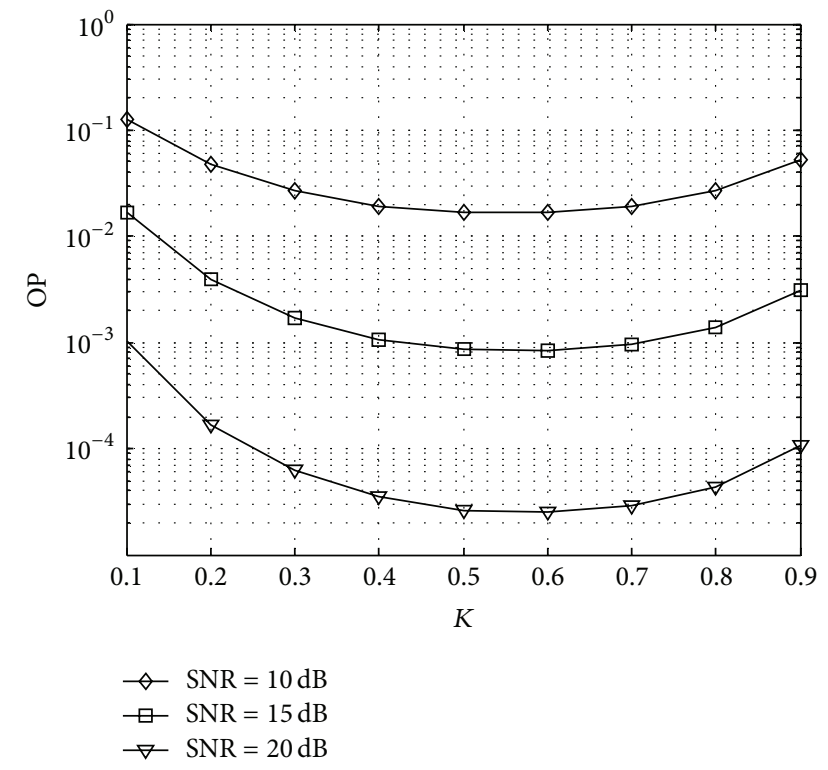

FIgURE 3: The effect of the power-allocation parameter $K$ on the OP performance.

optimal value of $K$ is approximately 0.6 , and for $\mathrm{SNR}=20 \mathrm{~dB}$, the optimal value of $K$ is also approximately 0.6.

Figure 4 presents the effect of the relative geometric gain $\mu$ on the OP performance of the M2M cooperative network over $N$-Nakagami fading channels. The number of cascaded components is $N=2$, and the fading coefficient is $m=2$. The relative geometric gains considered are $\mu=10 \mathrm{~dB}, 0 \mathrm{~dB}$, and $-10 \mathrm{~dB}$. The thresholds are $\gamma_{\mathrm{th}}=4 \mathrm{~dB}$ and $\gamma_{T}=2 \mathrm{~dB}$, and the power-allocation parameter is $K=0.5$. These results show that the OP performance is improved as $\mu$ is reduced. For example, when $\mathrm{SNR}=12 \mathrm{~dB}$ and $\mu=10 \mathrm{~dB}$, the OP is $2 \times$ $10^{-1}$, when $\mu=0 \mathrm{~dB}$ the OP is $3 \times 10^{-2}$, and when $\mu=-10 \mathrm{~dB}$,

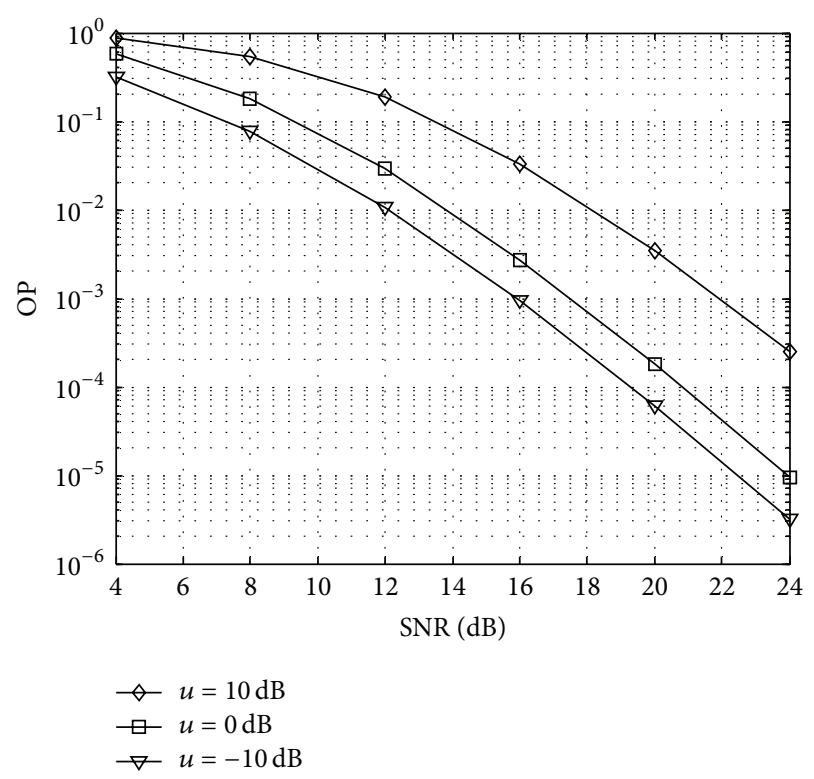

FIgURE 4: The effect of the relative geometric gain $\mu$ on the OP performance.

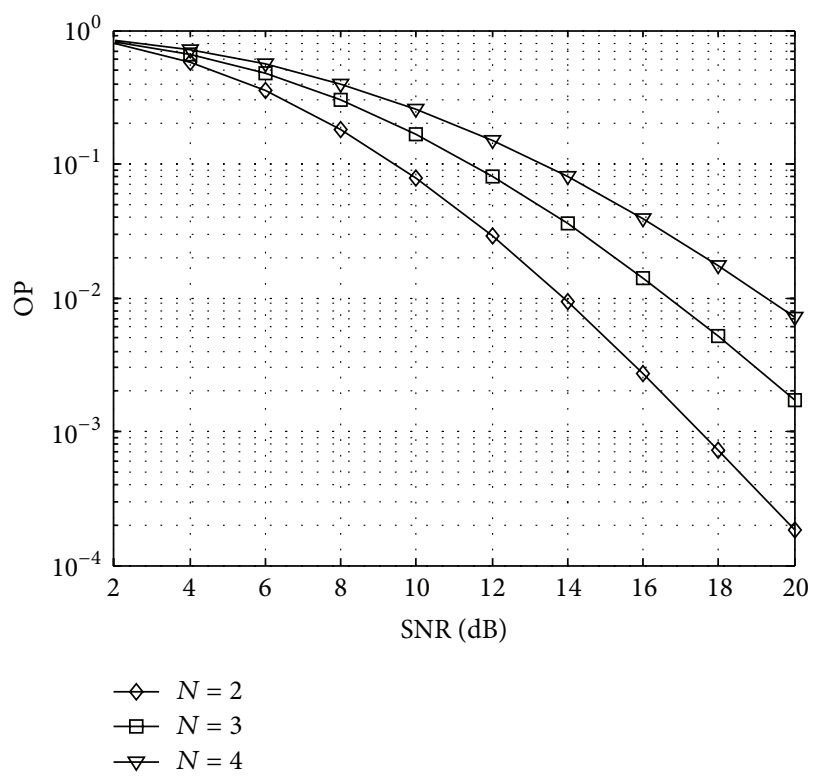

Figure 5: The effect of the number of cascaded components $N$ on the OP performance.

the OP is $1 \times 10^{-2}$. As the SNR increases, the OP gradually reduces.

Figure 5 presents the effect of the number of cascaded components $N$ on the OP performance of the M2M cooperative network over $N$-Nakagami fading channels. The number of cascaded components is $N=2,3,4$, which denote 2Nakagami, 3-Nakagami, and 4-Nakagami fading channels, respectively. The fading coefficient is $m=2$, the relative geometric gain is $\mu=0 \mathrm{~dB}$, and the thresholds are $\gamma_{\text {th }}=4 \mathrm{~dB}$ 
and $\gamma_{T}=2 \mathrm{~dB}$. The power-allocation parameter is $K=0.5$. These results show that the OP performance is degraded as $N$ is increased. For example, when $\mathrm{SNR}=12 \mathrm{~dB}$ and $N=2$, the OP is $3 \times 10^{-2}$, when $N=3$ the OP is $8 \times 10^{-2}$, and when $N=4$ the OP is $1.5 \times 10^{-1}$. This is because the fading severity for the cascaded channels increases as $N$ is increased. For fixed $N$, an increase in the SNR reduces the OP gradually.

\section{Conclusion}

A lower bound on the outage probability (OP) of SNRbased HDAF M2M cooperative network over $N$-Nakagami fading channels was derived. Performance results were presented which show that the fading coefficient $m$, number of cascaded components $N$, relative geometric gain $\mu$, and power-allocation parameter $K$ have a significant influence on the OP. The expressions derived in this paper are simple to compute and thus complete and accurate performance results can easily be obtained with minimal computational effort. In the future, the impact of correlated channels on the OP performance of M2M cooperative networks can be considered.

\section{Appendix}

Equation (27) can be simplified as follows:

$$
\begin{aligned}
Q= & \int_{0}^{\gamma_{\mathrm{th}}}\left(\int_{0}^{\gamma_{T}} f_{\gamma_{\mathrm{SR}}}(y) d y-\int_{0}^{\gamma_{\mathrm{RD}}} f_{\gamma_{\mathrm{SR}}}(y) d y\right) f_{\gamma_{\mathrm{RD}}}(z) d z \\
& +\int_{0}^{\gamma_{\mathrm{th}}}\left(\int_{0}^{\infty} f_{\gamma_{\mathrm{RD}}}(y) d y-\int_{0}^{\gamma_{\mathrm{SR}}} f_{\gamma_{\mathrm{RD}}}(y) d y\right) f_{\gamma_{\mathrm{SR}}}(z) d z \\
= & \int_{0}^{\gamma_{\mathrm{th}}} \int_{0}^{\gamma_{T}} f_{\gamma_{\mathrm{SR}}}(y) d y f_{\gamma_{\mathrm{RD}}}(z) d z \\
& -\int_{0}^{\gamma_{\mathrm{th}}} \int_{0}^{\gamma_{\mathrm{RD}}} f_{\gamma_{\mathrm{SR}}}(y) d y f_{\gamma_{\mathrm{RD}}}(z) d z \\
& +\int_{0}^{\gamma_{\mathrm{th}}} \int_{0}^{\infty} f_{\gamma_{\mathrm{RD}}}(y) d y f_{\gamma_{\mathrm{SR}}}(z) d z \\
& -\int_{0}^{\gamma_{\mathrm{th}}} \int_{0}^{\gamma_{\mathrm{SR}}} f_{\gamma_{\mathrm{RD}}}(y) d y f_{\gamma_{\mathrm{SR}}}(z) d z \\
= & A-B+C-D .
\end{aligned}
$$

First consider part $A$, which is given by

$$
\begin{aligned}
& \int_{0}^{\gamma_{T}} f_{\gamma_{\mathrm{SR}}}(y) d y \\
& \quad=\int_{0}^{\gamma_{T}} \frac{1}{y \prod_{t=1}^{N} \Gamma\left(m_{t}\right)} G_{0, N}^{N, 0}\left[\left.\frac{y}{\overline{\gamma_{\mathrm{SR}}}} \prod_{t=1}^{N} \frac{m_{t}}{\Omega_{t}}\right|_{m_{1}, \ldots, m_{N}} ^{-}\right] d y .
\end{aligned}
$$

To evaluate the integral in (A.2), the following integral function can be employed [13]:

$$
\begin{aligned}
& \int_{0}^{y} x^{a-1} G_{p, q}^{m, n}\left[\left.w x\right|_{b_{1}, \ldots, b_{q}} ^{a_{1}, \ldots, a_{p}}\right] d x \\
& \quad=y^{a} G_{p+1, q+1}^{m, n+1}\left[\left.w y\right|_{b_{1}, \ldots, b_{m},-a, b_{m+1}, \ldots, b_{q}} ^{a_{1}, \ldots, a_{n}, 1-a, a_{n}, \ldots, a_{p}}\right] .
\end{aligned}
$$

Equation (A.2) can then be expressed as

$$
\begin{aligned}
\int_{0}^{\gamma_{T}} f_{\gamma_{\mathrm{SR}}}(y) d y= & \frac{1}{\prod_{t=1}^{N} \Gamma\left(m_{t}\right)} \\
& \times G_{1, N+1}^{N, 1}\left[\left.\frac{\gamma_{T}}{\overline{\gamma_{\mathrm{SR}}}} \prod_{t=1}^{N} \frac{m_{t}}{\Omega_{t}}\right|_{m_{1}, \ldots, m_{N}, 0} ^{1}\right],
\end{aligned}
$$

so that $A$ is given by

$$
\begin{aligned}
A= & \frac{1}{\prod_{t=1}^{N} \Gamma\left(m_{t}\right) \prod_{t t=1}^{N} \Gamma\left(m_{t t}\right)} \\
& \times G_{1, N+1}^{N, 1}\left[\left.\frac{\gamma_{T}}{\overline{\gamma_{\mathrm{SR}}}} \prod_{t=1}^{N} \frac{m_{t}}{\Omega_{t}}\right|_{m_{1}, \ldots, m_{N}, 0} ^{1}\right] \\
& \times G_{1, N+1}^{N, 1}\left[\left.\frac{\gamma_{\mathrm{th}}}{\overline{\gamma_{\mathrm{RD}}}} \prod_{t t=1}^{N} \frac{m_{t t}}{\Omega_{t t}}\right|_{m_{1}, \ldots, m_{N}, 0} ^{1}\right] .
\end{aligned}
$$

Next, consider part $B$. Following a procedure similar to that for (A.2) yields

$$
\begin{aligned}
\int_{0}^{\gamma_{\mathrm{RD}}} f_{\gamma_{\mathrm{SR}}}(y) d y= & \frac{1}{\prod_{t=1}^{N} \Gamma\left(m_{t}\right)} \\
& \times G_{1, N+1}^{N, 1}\left[\left.\frac{\gamma_{\mathrm{RD}}}{\overline{\gamma_{\mathrm{SR}}}} \prod_{t=1}^{N} \frac{m_{t}}{\Omega_{t}}\right|_{m_{1}, \ldots, m_{N}, 0} ^{1}\right],
\end{aligned}
$$

so that $B$ is given by

$$
\begin{aligned}
B= & \int_{0}^{\gamma_{\mathrm{th}}} \int_{0}^{\gamma_{\mathrm{RD}}} f_{\gamma_{\mathrm{SR}}}(y) d y f_{\gamma_{\mathrm{RD}}}(z) d z \\
= & \frac{1}{\prod_{t=1}^{N} \Gamma\left(m_{t}\right) \prod_{t t=1}^{N} \Gamma\left(m_{t t}\right)} \\
& \times \int_{0}^{\gamma_{\mathrm{th}}} \frac{1}{z} G_{1, N+1}^{N, 1}\left[\left.\frac{z}{\overline{\gamma_{\mathrm{SR}}}} \prod_{t=1}^{N} \frac{m_{t}}{\Omega_{t}}\right|_{m_{1}, \ldots, m_{N}, 0} ^{1}\right] \\
& \times G_{0, N}^{N, 0}\left[\left.\frac{z}{\overline{\gamma_{\mathrm{RD}}}} \prod_{t t=1}^{N} \frac{m_{t t}}{\Omega_{t t}}\right|_{m_{1}, \ldots, m_{N}} ^{-}\right] d z .
\end{aligned}
$$

Next, consider part $C$. Since

$$
\int_{0}^{\infty} f_{\gamma_{\mathrm{RD}}}(y) d y=F_{\gamma_{\mathrm{RD}}}(\infty)=1
$$


$C$ can be expressed as

$$
C=\frac{1}{\prod_{t=1}^{N} \Gamma\left(m_{t}\right)} G_{1, N+1}^{N, 1}\left[\left.\frac{\gamma_{\mathrm{th}}}{\overline{\gamma_{\mathrm{SR}}}} \prod_{t=1}^{N} \frac{m_{t}}{\Omega_{t}}\right|_{m_{1}, \ldots, m_{N}, 0} ^{1}\right] .
$$

Finally, consider part $D$. Following a procedure similar to that for (A.2) yields

$$
\begin{aligned}
\int_{0}^{\gamma_{\mathrm{SR}}} f_{\gamma_{\mathrm{RD}}}(y) d y= & \frac{1}{\prod_{t t=1}^{N} \Gamma\left(m_{t t}\right)} \\
& \times G_{1, N+1}^{N, 1}\left[\left.\frac{\gamma_{\mathrm{SR}}}{\overline{\gamma_{\mathrm{RD}}}} \prod_{t t=1}^{N} \frac{m_{t t}}{\Omega_{t t}}\right|_{m_{1}, \ldots, m_{N}, 0} ^{1}\right],
\end{aligned}
$$

so that $D$ is given by

$$
\begin{aligned}
D= & \int_{0}^{\gamma_{\mathrm{th}}} \int_{0}^{\gamma_{\mathrm{SR}}} f_{\gamma_{\mathrm{RD}}}(y) d y f_{\gamma_{\mathrm{SR}}}(z) d z \\
= & \frac{1}{\prod_{t=1}^{N} \Gamma\left(m_{t}\right) \prod_{t t=1}^{N} \Gamma\left(m_{t t}\right)} \\
& \times \int_{0}^{\gamma_{\mathrm{th}}} \frac{1}{z} G_{1, N+1}^{N, 1}\left[\left.\frac{z}{\overline{\gamma_{\mathrm{RD}}}} \prod_{t t=1}^{N} \frac{m_{t t}}{\Omega_{t t}}\right|_{m_{1}, \ldots, m_{N}, 0} ^{1}\right] \\
& \times G_{0, N}^{N, 0}\left[\left.\frac{z}{\overline{\gamma_{\mathrm{SR}}}} \prod_{t=1}^{N} \frac{m_{t}}{\Omega_{t}}\right|_{m_{1}, \ldots, m_{N}} ^{-}\right] d z .
\end{aligned}
$$

\section{Conflict of Interests}

The authors declare that there is no conflict of interests regarding the publication of this paper.

\section{Acknowledgments}

The authors would like to thank the referees and editor for providing helpful comments and suggestions. This project was supported by the National Natural Science Foundation of China (no. 61304222 and no. 60902005), the Natural Science Foundation of Shandong Province (no. ZR2012FQ021), the Shandong Province Higher Educational Science and Technology Program (no. J12LN88), and the International Science and Technology Cooperation Program of Qingdao (no. 12-14-137-hz).

\section{References}

[1] S. Mumtaz, K. M. Saidul Huq, and J. Rodriguez, "Direct mobileto-mobile communication: paradigm for 5G," IEEE Wireless Communications, vol. 21, no. 5, pp. 14-23, 2014.

[2] L. Z. Xun, H. H. Ying, R. X. Kun, and C. W. Kui, "Influences of double-Rayleigh transmission system performance," Journal of XiDian University, vol. 38, no. 5, pp. 172-177, 2011.

[3] H. Ilhan, I. Altunbaş, and M. Uysal, "Moment generating function-based performance evaluation of amplify-andforward relaying in $\mathrm{N}^{*}$ Nakagami fading channels," IET Communications, vol. 5, no. 3, pp. 253-263, 2011.
[4] G. K. Karagiannidis, N. C. Sagias, and P. T. Mathiopoulos, " $\mathrm{N}^{*}$ Nakagami: a novel stochastic model for cascaded fading channels," IEEE Transactions on Communications, vol. 55, no. 8, pp. 1453-1458, 2007.

[5] H. Ilhan, M. Uysal, and I. Altunbaş, "Cooperative diversity for intervehicular communication: performance analysis and optimization," IEEE Transactions on Vehicular Technology, vol. 58, no. 7, pp. 3301-3310, 2009.

[6] F. K. Gong, J. Ge, and N. Zhang, "SER analysis of the mobilerelay-based M2M communication over double Nakagami-m fading channels," IEEE Communications Letters, vol. 15, no. 1, pp. 34-36, 2011.

[7] F. K. Gong, P. Ye, Y. Wang, and N. Zhang, "Cooperative mobileto-mobile communications over double Nakagami-m fading channels," IET Communications, vol. 6, no. 18, pp. 3165-3175, 2012.

[8] T. Q. Duong and H.-J. Zepernick, "On the performance gain of hybrid decode-amplify-forward cooperative communications," Eurasip Journal on Wireless Communications and Networking, vol. 2009, Article ID 479463, 10 pages, 2009.

[9] H. Chen, J. Liu, C. Zhai, and L. Zheng, "Performance analysis of SNR-based hybrid decode-amplify-forward cooperative diversity networks over rayleigh fading channels," in Proceedings of the IEEE Wireless Communications and Networking Conference, pp. 1-6, Sydney, Australia, April 2010.

[10] W. F. Su, A. K. Sadek, and K. J. R. Liu, "Cooperative communication protocols in wireless networks: performance analysis and optimum power allocation," Wireless Personal Communications, vol. 44, no. 2, pp. 181-217, 2008.

[11] P. A. Anghel and M. Kaveh, "Exact symbol error probability of a cooperative network in a rayleigh-fading environment," IEEE Transactions on Wireless Communications, vol. 3, no. 5, pp. 14161421, 2004.

[12] A. Papoulis and S. U. Pillai, Random Variables and Stochastic Processes, McGraw-Hill, New York, NY, USA, 4th edition, 2002.

[13] A. M. Mathai and R. K. Saxena, Generalized Hypergeometric Functions with Applications in Statistics and Physical Sciences, Springer, New York, NY, USA, 1973. 

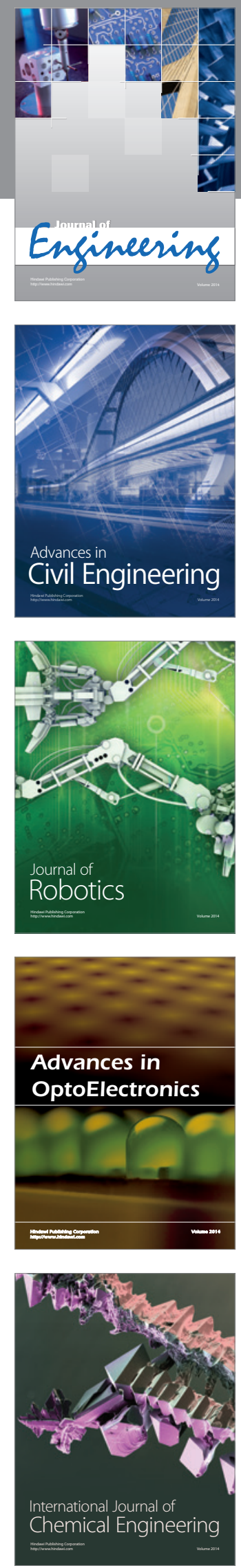

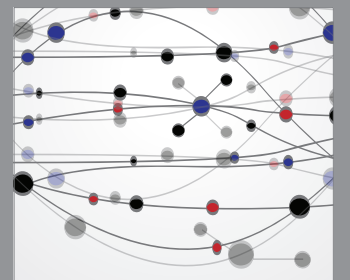

The Scientific World Journal
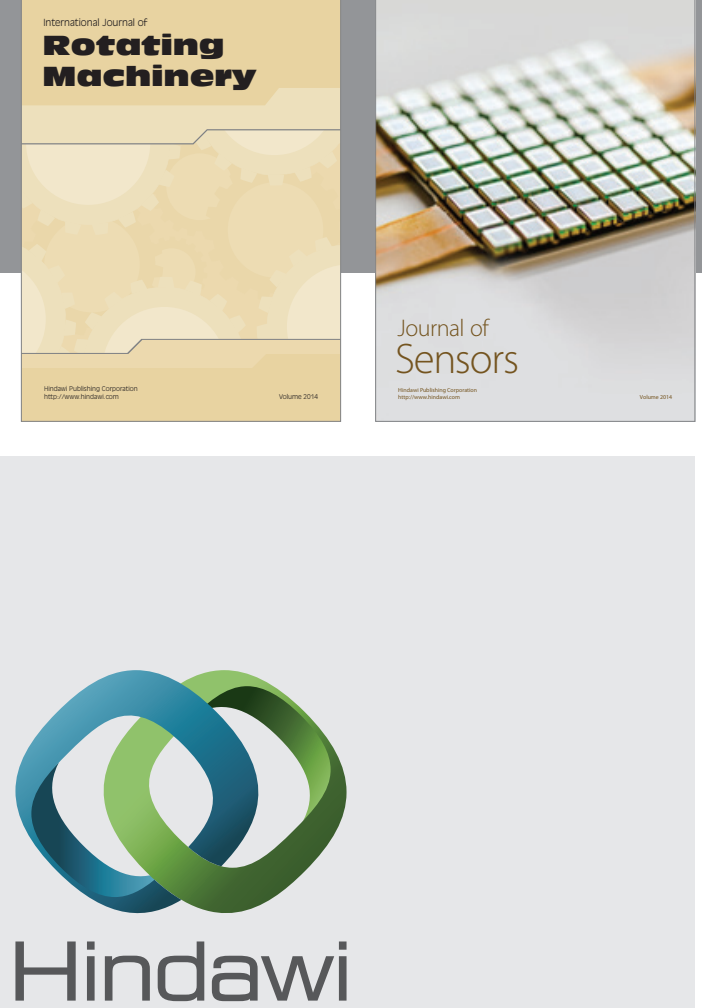

Submit your manuscripts at http://www.hindawi.com
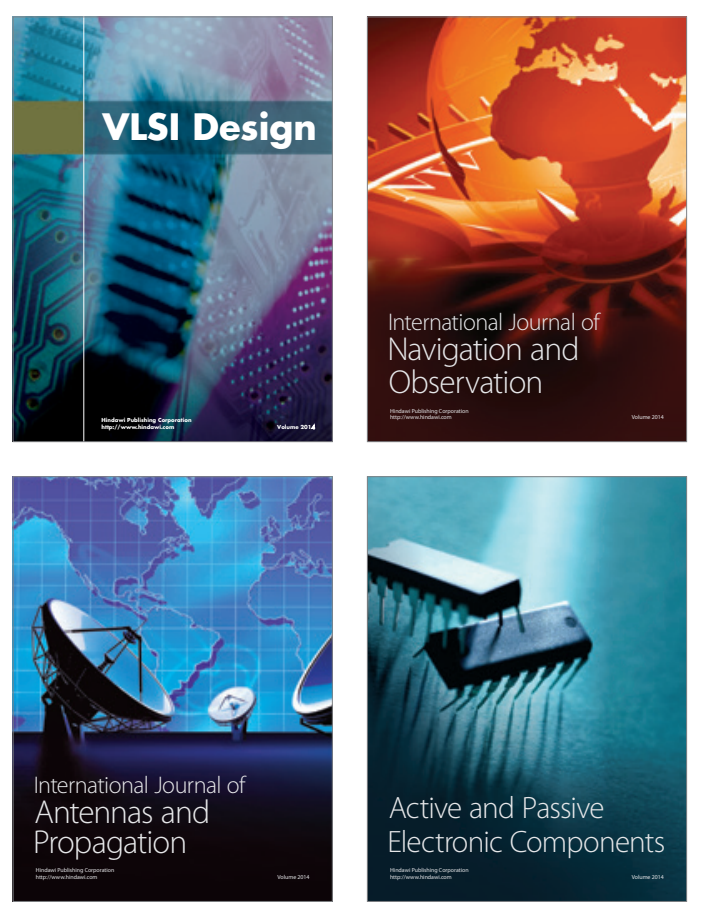
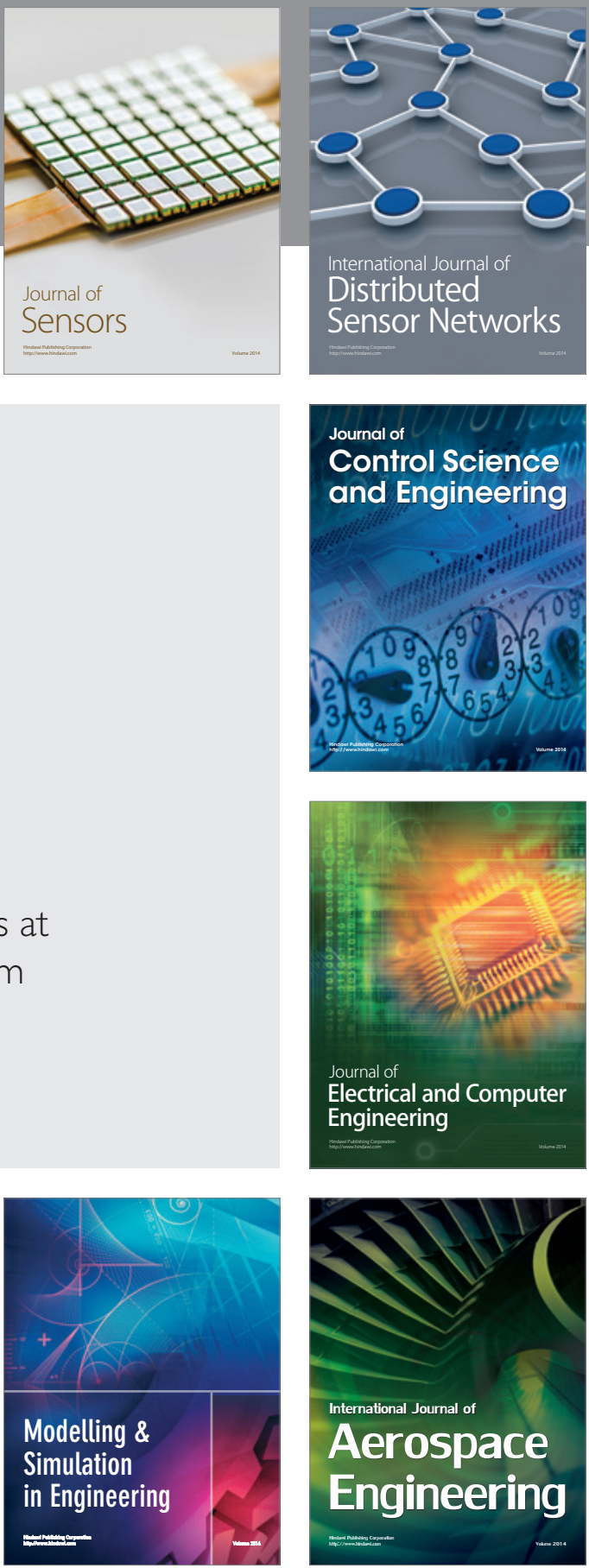

Journal of

Control Science

and Engineering
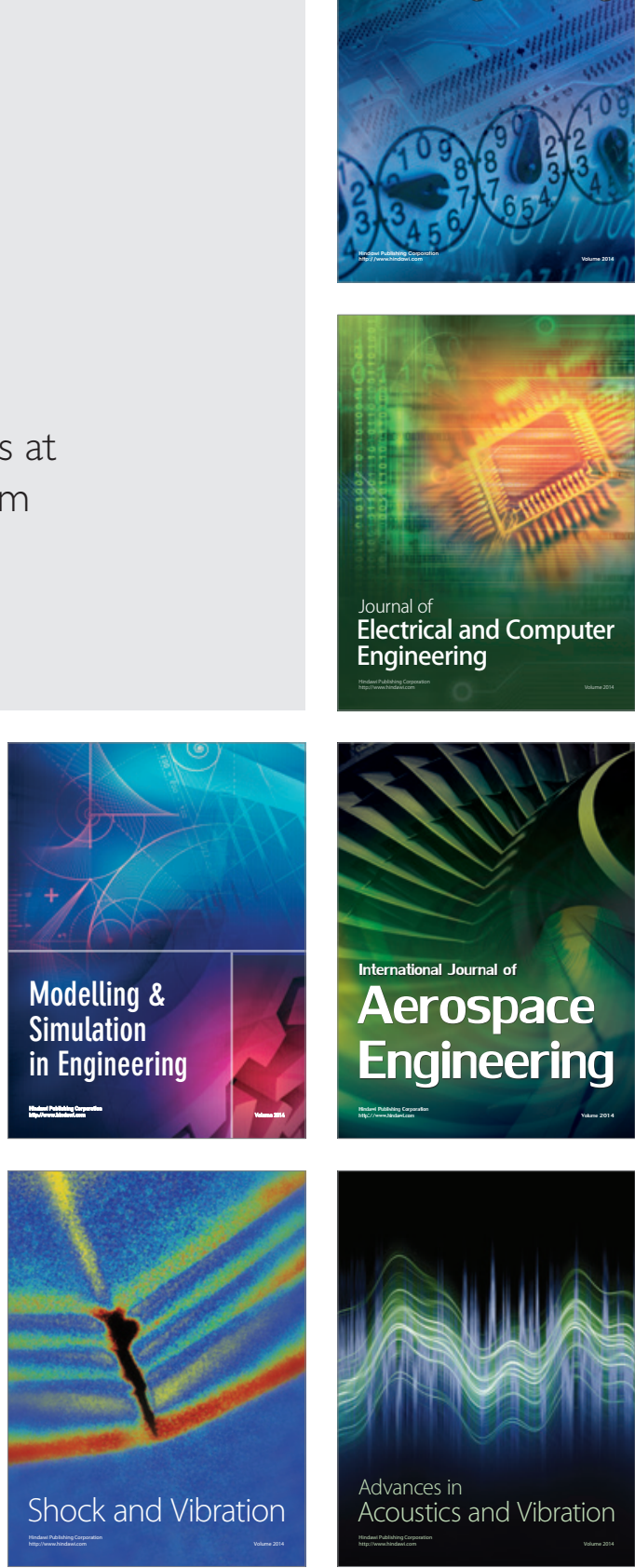